\title{
Chaos Control in a Discrete Ecological System
}

\author{
Limin Zhang*, Chaofeng Zhang \\ Department of Mathematics and Finance-Economics, Sichuan University of Arts and Science, Dazhou, China \\ Email: lmzhang2000@163.com
}

Received August 23, 2012; revised September 7, 2012; accepted September 14, 2012

\begin{abstract}
In research [1], the authors investigate the dynamic behaviors of a discrete ecological system. The period-double bifurcations and chaos are found in the system. But no strategy is proposed to control the chaos. It is well known that chaos control is the first step of utilizing chaos. In this paper, a controller is designed to stabilize the chaotic orbits and enable them to be an ideal target one. After that, numerical simulations are presented to show the correctness of theoretical analysis.
\end{abstract}

Keywords: Chaos Control; Discrete Ecological System; Numerical Simulation

\section{Introduction}

Population dynamics in ecology are generally governed by discrete and continuous systems. In recent years, the study of discrete ecological systems has attracted extensive attentions [1-6]. This is because that some natural populations have non-overlapping generations, thus discrete models are more realistic than continuous ones to study these species. Another reason is that people always study population changes by one year (mouth, week or day). Such investigations are often required discrete models. Especially, using discrete models is more efficient for numerical simulations. Recently, Zhang and Li [1] studied the following discrete ecological model:

$$
\begin{gathered}
x_{n+1}=x_{n}+\delta\left(1-x_{n}+x_{n} y_{n}\right) \\
y_{n+1}=y_{n}+\delta\left(a-b x_{n}\right) y_{n}^{2}
\end{gathered}
$$

where $x_{n}, y_{n}$ denote the two ecological species' densities respectively in generation $n ; \delta$ is the integral step size. The more meaning of system (1) can refer to the reference $[1,2]$. It is shown that the system (1) generates period-double bifurcations and chaos. But the authors did not investigate the chaos control of the system.

It is well known that chaos control is the first step of utilizing chaos. The possibility of chaos control in biological systems has been stimulated by recent advances in the study of heart and brain tissue dynamics. Recently, some authors have investigated that such a method can be applied to population dynamics and even play a nontrivial evolutionary role in ecology [7-9]. In this paper, we design a proper controller to control the chaos of system (1).

\section{Chaos Control}

In this section, chaotic orbits to an unstable fixed point are stabilized by utilizing some control techniques. Firstly, we introduce the following lemma which is useful to establish our results

Lemma 1 [1]. If $a>b$, then system (1) has an unique positive fixed point at $E\left(x^{*}, y^{*}\right)$, where $x^{*}=a / b$, $y^{*}=1-b / a$.

Consider the following map which is the feedback is applied to system (1)

$$
X_{n+1}=F\left(X_{n}, \mu_{n}\right)=\left(\begin{array}{l}
x_{n}+\delta\left(1-x_{n}+x_{n} y_{n}\right)+\mu_{n} \\
y_{n}+\delta\left(a-b x_{n}\right) y_{n}^{2}
\end{array}\right)
$$

where $X_{n}=\left(x_{n}, y_{n}\right)^{T}, \mu_{n}$ is control variable and satisfies $\left|\mu_{n}\right|<\varepsilon, \quad \varepsilon \square 1$. Evidently, map (2) degenerates to original system (1) only if $\mu_{n}=0$. We select the feedback variable $\mu_{n}$ in the range $(-\varepsilon, \varepsilon)$, so that the orbit holds in the neighborhood of fixed point $E$ as long as the control arises. The ergodic nature of the chaotic dynamics guarantees that the mode trajectory in the neighborhood of the wishful orbit $E\left(x^{*}, y^{*}\right)$. In the neighborhood of $E$, map (2) can be approximated by the following form:

$$
\left(\begin{array}{c}
x_{n+1}-x^{*} \\
y_{n+1}-y^{*}
\end{array}\right) \rightarrow A\left(\begin{array}{c}
x_{n}-x^{*} \\
y_{n}-y^{*}
\end{array}\right)+B \mu_{n}
$$

where $A$ is the Jacobian matrix at $E$ and $B$ is a column vector, and they are given by:

$$
A=\left.\frac{\partial F}{\partial X_{n}}\right|_{X_{n}=E, \mu_{n}=0}=\left(\begin{array}{cc}
1-\frac{\delta b}{a} & \frac{\delta a}{b} \\
-\delta b\left(1-\frac{b}{a}\right)^{2} & 1
\end{array}\right),
$$




$$
B=\left.\frac{\partial F}{\partial \mu_{n}}\right|_{X_{n}=E, \mu_{n}=0}=\left(\begin{array}{l}
1 \\
0
\end{array}\right) .
$$

Let $X^{*}=\left(x^{*}, y^{*}\right)^{T}$ and suppose that $\mu_{n}$ is a linear function of $X_{n}$, which is expressed as $\mu_{n}=P^{T}\left(X n-X^{*}\right)$, $P^{T} \in R^{1 \times 2}$. Substitute the result into (3), we get

$$
\left(\begin{array}{c}
x_{n+1}-x^{*} \\
y_{n+1}-y^{*}
\end{array}\right) \rightarrow\left(A-B P^{T}\right)\left(\begin{array}{c}
x_{n}-x^{*} \\
y_{n}-y^{*}
\end{array}\right) .
$$

According to the study [10], the fixed point $E$ will be stable if the matrix $\left(A-B P^{T}\right)$ is asymptotically stable, that is to say, all its eigenvalues are less than 1 in modulus. Now, we make use of "pole placement technique" [11] to determine the specific values in $\left(A-B P^{T}\right)$. If system (1) is chaotic, we obtain

$$
\left|1-\frac{\delta b}{2 a}\right|>1
$$

Then we choose

$$
\sigma_{1}=0, \sigma_{2}=1-\frac{\delta b}{2 a}
$$

as the desired eigenvalues of the matrix $\left(A-B P^{T}\right)$. The controllability matrix

$$
C=\left(\begin{array}{ll}
B & A B
\end{array}\right)=\left(\begin{array}{cc}
1 & 1-\frac{\delta b}{a} \\
0 & -\delta b\left(1-\frac{b}{a}\right)^{2}
\end{array}\right)
$$

has two rank. Thus the solution to the pole placement problem is obtained as

$$
P^{T}=\left(q_{2}-p_{2} \quad q_{1}-p_{1}\right) Q^{-1},
$$$$
\text { where } \quad Q=C W, W=\left(\begin{array}{cc}
p_{1} & 1 \\
1 & 0
\end{array}\right) \text {, }
$$

$p_{1}$ and $p_{2}$ are the coefficients of characteristic polynomial of the matrix $A, \operatorname{det}(\lambda I-A)=\lambda^{2}+p_{1} \lambda+p_{2}$,

$$
\text { and } \quad p_{1}=-2+\frac{\delta a}{b}, p_{2}=\frac{\delta^{2}(a-b)^{2}-\delta b}{a} ;
$$

$q_{1}$ and $q_{2}$ are the coefficients of characteristic polynomial of the matrix $\left(A-B P^{T}\right)$,

$$
\operatorname{det}\left(\sigma I-\left(A-B P^{T}\right)\right)=\lambda^{2}+q_{1} \lambda+q_{2},
$$

and $\quad q_{1}=\frac{\delta b}{2 a}-1, q_{2}=0$.

After calculations, we get

$$
P^{T}=\left(\frac{2 a-\delta b}{2 a} \frac{a\left[a-\delta b+\delta^{2}(a-b)^{2}\right]}{\delta b(a-b)^{2}}+\frac{a(2 a-\delta b)}{2 \delta b(a-b)^{2}}\right)
$$

Furthermore, the controller has the following form:

$$
\mu_{n}=P^{T}\left(\begin{array}{ll}
\varepsilon x_{n} & \varepsilon y_{n}
\end{array}\right)^{T},
$$

where $\varepsilon x_{n}=x_{n}-x^{*}, \varepsilon y_{n}=y_{n}-y^{*}$.

However, the above considerations only are fit for a local small neighbor of $E$. In view of the global situation, we can specify $\mu_{n}$ by making $\mu_{n}=0$ if $\left|P^{T}\left(X_{n}-X^{*}\right)\right|$ is too large. This is because the range of $\mu_{n}$ is restrained by $\left|\mu_{n}\right|<\varepsilon$ and $\varepsilon \square 1$. Thus, we limit the number value

$$
\left|X_{n}-X^{*}\right|<\frac{\varepsilon}{\left|P^{T}\right|} .
$$

Therefore, in practice we take $\mu_{n}$ as

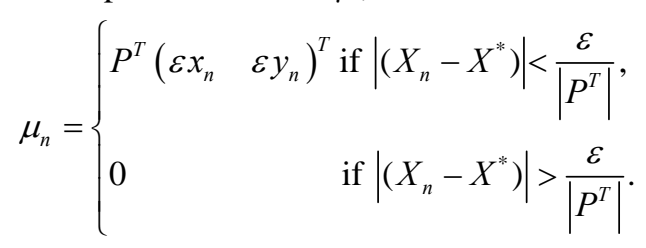

According to the above analysis, we get the following result.

Theorem 1. If

$$
\left|\left(X_{n}-X^{*}\right)\right|<\frac{\varepsilon}{\left|P^{T}\right|},
$$

then the control variable $\mu_{n}=P^{T}\left(\begin{array}{ll}\varepsilon x_{n} & \varepsilon y_{n}\end{array}\right)^{T}$ can stabilize chaotic trajectory of system (1) to the fixed point $E$, where $P^{T}$ is given by Equation (4).

\section{Numerical Simulations}

In the section, we use density-time diagrams and phase portraits to confirm the above theoretical analysis.

Let $a=2.21, b=1.02, \delta=0.9666$. At the condition, $\left|P^{T}\right|$ has the value 7.70732 . According to Lemma 1, system (1) has and only has a positive fixed point $E\left(x^{*}, y^{*}\right)=$ (2.16667, 0.53846). We adopt

$$
B_{r}(E)=\left\{\left(x_{n}, y_{n}\right) \mid\left(x_{n}-x^{*}\right)^{2}+\left(y_{n}-y^{*}\right)^{2} \leq(0.001)^{2}\right\} \text {. }
$$

When $\varepsilon$ is given the value 0.03 and 0.09 , Theorem 1 is satisfied. Density-time diagram of ecological specie $x_{n}$ is given by Figure 1(a), which is characterized by switches between apparently regular and chaotic behaviors. Actually, it is intermittency, which is a basic characteristic of chaos. At the same parameters, phase portrait is illustrated by Figure 1(b), which is a chaotic attractor. Figure 2 is the chaos control diagrams corresponding to Figure 1. With the same parameters of Figure 1, system (1) is chaotic if $n<800$ when $\varepsilon=0.03$ (Figures 2(a) and (b)) according to the control strategy. Actually, Figure 2(a) is supertransient, which is used to denote an unusually long convergence to an attractor. Figure 2(b) is phase portrait corresponding to Figure 2(a). When $\varepsilon$ increases to 0.09 , supertransient disappears and the system 


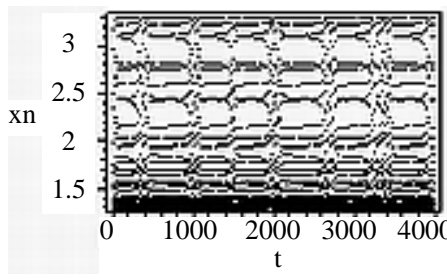

(a)

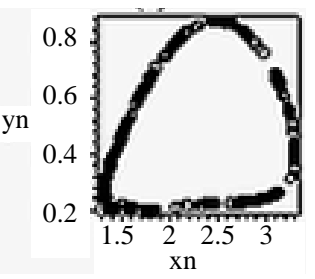

(b)
Figure 1. (a) Density-time diagram of $x_{n}$; (b) phase portrait of $x_{n}$ and $y_{n}$. Where the parameters given by $a=2.21, b=$ 1.02, $\delta=0.9666$.

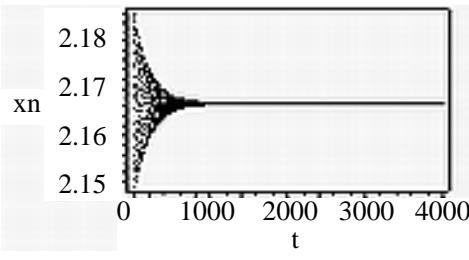

(a)

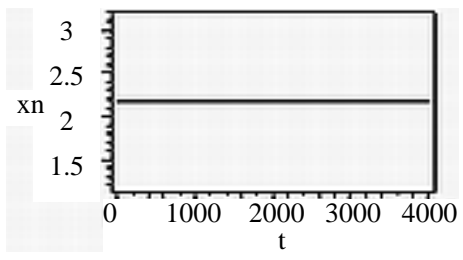

(c)

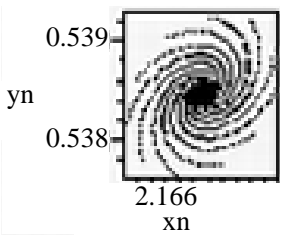

(b)

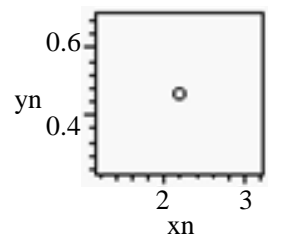

(d)
Figure 2. (a) Density-time controlled diagram of $x_{n}$ with $\varepsilon=$ 0.03; (b) controlled phase portrait with $\varepsilon=0.03$; (c) Density-time controlled diagram of $x_{n}$ with $\varepsilon=0.09$; (d) controlled phase portrait with $\varepsilon=0.09$. The other parameters given by $a=2.21, b=1.02, \delta=0.9666$.

stabilizes to the fixed point $(2.16667,0.53846)$, which is simulated by Figures 2(c) and 2(d).

\section{Conclusion}

In this paper, we design a proper controller to control the chaos of system (1) which was firstly studied by Zhang and $\mathrm{Li}$ [6]. From the theoretical analysis, we concluded that the control variable $\mu_{n}=P^{T}\left(\begin{array}{lll}\varepsilon x_{n} & \varepsilon y_{n}\end{array}\right)^{T}$ can stabilize chaotic trajectory of system (1) to the fixed point $E\left(x^{*}, y^{*}\right)$ under the condition of

$$
\left|\left(X_{n}-X^{*}\right)\right|<\frac{\varepsilon}{\left|P^{T}\right|},
$$

where $P^{T}$ is given by Equation (4). Then simulations are presented to show the correctness of theoretical analysis. Figure 1 demonstrates system (1) is chaotic with parameters $a=2.21, b=1.02, \delta=0.9666$. Figure 2 indicates system (1) processes from supertransient to the fixed point when the control variable applied to the system.

\section{Acknowledgements}

This work is supported by the National Natural Science Foundation of China (No. 30970305), the Sichuan Provincial Natural Science Foundation (No. 10ZB136), the Sichuan Provincial Old Revolutionary Base Areas Foundation (No. SLQ2010C-17).

\section{REFERENCES}

[1] L. M. Zhang and L. Li, "Dynamic Complexities in a Discrete Predator-Prey System," Journal of Wuhan University of Science and Engineering, Vol. 23, No. 3, 2010, pp. 36-40.

[2] L. M. Zhang, "Stability and Bifurcation in a Discrete Predator-Prey System with Leslie-Gower Type," Sichuan University of Arts and Science Journal, Vol. 20, No. 2, 2010, pp. 13-15.

[3] C. Celik and O. Duman, "Allee Effect in a Discrete-Time Predator-Prey System,” Chaos, Solitons \& Fractals, Vol. 40, No. 4, 2009, pp. 1956-1962. doi:10.1016/j.chaos.2007.09.077

[4] H. N. Agiza and E. M. Elabbssy, "Chaotic Dynamics of a Discrete Prey-Predator Model with Holling Type II," Nonlinear Analysis: Real World Applications, Vol. 10, No. 1, 2009, pp. 116-129. doi:10.1016/j.nonrwa.2007.08.029

[5] X. L. Liu and D. M. Xiao, "Complex Dynamic Behaviors of a Discrete-Time Predator-Prey System,” Chaos, Solitons \& Fractals, Vol. 32, No. 1, 2007, pp. 80-94. doi:10.1016/j.chaos.2005.10.081

[6] Z. J. Jing and J. P. Yang, "Bifurcation and Chaos in Discrete-Time Predator-Prey System," Chaos, Solitons \& Fractals, Vol. 27, No. 1, 2006, pp. 259-277. doi:10.1016/j.chaos.2005.03.040

[7] V. S. Ricard and G. P. G. Javier, "Controlling Chaos in Ecology: From Deterministic to Individual-Based Models,” Bulletin of Mathematical Biology, Vol. 61, No. 6, 1999, pp. 1187-1207. doi:10.1006/bulm.1999.0141

[8] Y. Zhang, Q. L. Zhang, L. C. Zhao and C. Y. Yang, “Dynamical Behaviors and Chaos Control in a Discrete Functional Response Model,” Chaos, Solitons and Fractals, Vol. 34, No. 4, 2007, pp. 1318-1327. doi:10.1016/j.chaos.2006.04.032

[9] A. A. Gomes, E. Manica, M. C. Varriale, “Applications of Chaos Control Techniques to a Three-Species Food Chain,” Chaos, Solitons \& Fractals, Vol. 36, No. 4, pp. 1097-1107. doi:10.1016/j.chaos.2006.07.027

[10] S. Boccaletti, C. Grebogi, Y.-C. Lai, H. Mancin and D. Maza, "The Control of Chaos: Theory and Applications," Physical Reports, Vol. 3, 2003, pp. 29-97.

[11] R. Thoresten, S. Alexander, U. Dressler, D. Robet, H. Bernd and M. Werner, "Chaos Control with Adjustable Control Times,” Chaos, Solitons \& Fractals, Vol. 8, No. 9, 1997, pp. 1559-1576. doi:10.1016/S0960-0779(96)00155-5 